\title{
Characterizing the $\gamma$-ray long-term variability of PKS 2155-304 with H.E.S.S. and Fermi-LAT
}

\begin{abstract}
H.E.S.S. Collaboration, H. Abdalla ${ }^{1}$, A. Abramowski ${ }^{2}$, F. Aharonian ${ }^{3,4,5}$, F. Ait Benkhali ${ }^{3}$, A. G. Akhperjanian ${ }^{6,5}$, $^{\dagger}$, T. Andersson ${ }^{10}$, E. O. Angüner ${ }^{7}$, M. Arrieta ${ }^{15}$, P. Aubert ${ }^{23}$, M. Backes ${ }^{8}$, A. Balzer ${ }^{9}$, M. Barnard ${ }^{1}$, Y. Becherini ${ }^{10}$, J. Becker Tjus ${ }^{11}$, D. Berge ${ }^{12}$, S. Bernhard $^{13}$, K. Bernlöhr ${ }^{3}$, R. Blackwell ${ }^{14}$, M. Böttcher ${ }^{1}$, C. Boisson ${ }^{15}$, J. Bolmont ${ }^{16}$, P. Bordas ${ }^{3}$, J. Bregeon ${ }^{17}$, F. Brun ${ }^{25}$, P. Brun ${ }^{18}$, M. Bryan ${ }^{9}$, T. Bulik ${ }^{19}$, M. Capasso ${ }^{27}$, J. Carr ${ }^{20}$, S. Casanova ${ }^{21,3}$, M. Cerruti ${ }^{16}$, N. Chakraborty ${ }^{3}$, R. Chalme-Calvet ${ }^{16}$, R. C. G. Chaves ${ }^{17}$, A. Chen ${ }^{22}$, J. Chevalier $^{23, \star}$, M. Chrétien ${ }^{16}$, S. Colafrancesco ${ }^{22}$, G. Cologna ${ }^{24}$, B. Condon ${ }^{25}$, J. Conrad ${ }^{26}$, Y. Cui ${ }^{27}$, I. D. Davids ${ }^{1,8}$, J. Decock ${ }^{18}$, B. Degrange ${ }^{28}$, C. Deil ${ }^{3}$,

J. Devin ${ }^{17}$, P. deWilt ${ }^{14}$, L. Dirson ${ }^{2}$, A. Djannati-Ataï ${ }^{29}$, W. Domainko ${ }^{3}$, A. Donath ${ }^{3}$, L. O'C. Drury ${ }^{4}$, G. Dubus ${ }^{30}$, K. Dutson ${ }^{31}$, J. Dyks $^{32}$, T. Edwards ${ }^{3}$, K. Egberts 3 , P. Eger ${ }^{3}$, J.-P. Ernenwein ${ }^{20}$, S. Eschbach ${ }^{34}$, C. Farnier ${ }^{26,10}$, S. Fegan ${ }^{28}$, M. V. Fernandes ${ }^{2}$, A. Fiasson ${ }^{23}$, G. Fontaine ${ }^{28}$, A. Förster ${ }^{3}$, S. Funk ${ }^{34}$, M. Füßling ${ }^{35}$, S. Gabici ${ }^{29}$, M. Gajdus ${ }^{7}$, Y. A. Gallant ${ }^{17}$, T. Garrigoux ${ }^{1}$, G. Giavitto ${ }^{35}$, B. Giebels ${ }^{28}$, J. F. Glicenstein ${ }^{18}$, D. Gottschall ${ }^{27}$, A. Goyal ${ }^{36}$, M.-H. Grondin ${ }^{25}$, D. Hadasch ${ }^{13}$, J. Hahn ${ }^{3}$, M. Haupt ${ }^{35}$, J. Hawkes ${ }^{14}$, G. Heinzelmann ${ }^{2}$, G. Henri ${ }^{30}$, G. Hermann ${ }^{3}$, O. Hervet ${ }^{15,42}$, J. A. Hinton ${ }^{3}$, W. Hofmann ${ }^{3}$, C. Hoischen ${ }^{33}$, M. Holler ${ }^{28}$, D. Horns ${ }^{2}$, A. Ivascenko ${ }^{1}$, A. Jacholkowska ${ }^{16}$, M. Jamrozy ${ }^{36}$, M. Janiak ${ }^{32}$, D. Jankowsky ${ }^{34}$, F. Jankowsky ${ }^{24}$, M. Jingo ${ }^{22}$, T. Jogler ${ }^{34}$, L. Jouvin ${ }^{29}$, I. Jung-Richardt ${ }^{34}$, M. A. Kastendieck ${ }^{2}$, * K. Katarzyński ${ }^{37}$, U. Katz ${ }^{34}$, D. Kerszberg ${ }^{16}$, B. Khélifi' ${ }^{29}$, M. Kieffer ${ }^{16}$, J. King ${ }^{3}$, S. Klepser ${ }^{35}$, D. Klochkov ${ }^{27}$, W. Kluźniak ${ }^{32}$, D. Kolitzus ${ }^{13}$, Nu. Komin ${ }^{22}$, K. Kosack ${ }^{18}$, S. Krakau ${ }^{11}$, M. Kraus ${ }^{34}$, F. Krayzel ${ }^{23}$, P. P. Krüger ${ }^{1}$, H. Laffon ${ }^{25}$, G. Lamanna ${ }^{23}$, J. Lau ${ }^{14}$, J.-P. Lees ${ }^{23}$, J. Lefaucheur ${ }^{15}$, V. Lefranc ${ }^{18}$, A. Lemière ${ }^{29}$, M. Lemoine-Goumard ${ }^{25}$, J.-P. Lenain ${ }^{16}$, E. Leser $^{33}$, T. Lohse ${ }^{7}$, M. Lorentz ${ }^{18}$, R. Liu ${ }^{3}$, R. López-Coto ${ }^{3}$, I. Lypova ${ }^{35}$, V. Marandon $^{3}$, A. Marcowith ${ }^{17}$, C. Mariaud ${ }^{28}$, R. Marx ${ }^{3}$, G. Maurin ${ }^{23}$, N. Maxted $^{14}$, M. Mayer ${ }^{7}$, P. J. Meintjes ${ }^{38}$, M. Meyer ${ }^{26}$, A. M. W. Mitchell ${ }^{3}$, R. Moderski ${ }^{32}$, M. Mohamed ${ }^{24}$, L. Mohrmann ${ }^{34}$, K. Morä ${ }^{26}$, E. Moulin ${ }^{18}$, T. Murach ${ }^{7}$, M. de Naurois ${ }^{28}$, F. Niederwanger ${ }^{13}$, J. Niemiec ${ }^{21}$, L. Oakes $^{7}$, P. O'Brien ${ }^{31}$, H. Odaka ${ }^{3}$, S. Ött1 ${ }^{13}$, S. Ohm ${ }^{35}$, M. Ostrowski ${ }^{6}$, I. Oya ${ }^{35}$, M. Padovani ${ }^{17}$, M. Panter ${ }^{3}$, R. D. Parsons ${ }^{3}$, N. W. Pekeur ${ }^{1}$, G. Pelletier $^{30}$, C. Perennes $^{16}$, P.-O. Petrucci ${ }^{30}$, B. Peyaud ${ }^{18}$, Q. Piel ${ }^{23}$, S. Pita ${ }^{29}$, H. Poon ${ }^{3}$, D. Prokhorov ${ }^{10}$, H. Prokoph ${ }^{10}$, G. Pühlhofer ${ }^{27}$, M. Punch ${ }^{29,10}$, A. Quirrenbach ${ }^{24}$, S. Raab ${ }^{34}$, A. Reimer ${ }^{13}$, O. Reimer ${ }^{13}$, M. Renaud ${ }^{17}$, R. de los Reyes ${ }^{3}$, F. Rieger ${ }^{3,39, *}$, C. Romoli ${ }^{4}$, S. Rosier-Lees ${ }^{23}$, G. Rowell $^{14}$, B. Rudak $^{32}$,

C. B. Rulten ${ }^{15}$, V. Sahakian ${ }^{6,5}$, D. Salek ${ }^{40}$, D. A. Sanchez ${ }^{23}$, A. Santangelo ${ }^{27}$, M. Sasaki ${ }^{27}$, R. Schlickeiser ${ }^{11}$, F. Schüssler ${ }^{18}$, A. Schulz ${ }^{35}$, U. Schwanke ${ }^{7}$, S. Schwemmer ${ }^{24}$, M. Settimo ${ }^{16}$, A. S. Seyffert ${ }^{1}$, N. Shafi ${ }^{22}$, I. Shilon ${ }^{34}$, R. Simoni ${ }^{9}$, H. Sol ${ }^{15}$, F. Spanier ${ }^{1}$, G. Spengler $^{26}$, F. Spies $^{2}$, Ł. Stawarz ${ }^{36}$, R. Steenkamp ${ }^{8}$, C. Stegmann ${ }^{33,35}$, F. Stinzing ${ }^{34}$, , K. Stycz ${ }^{35}$, I. Sushch ${ }^{1}$, J.-P. Tavernet ${ }^{16}$, T. Tavernier ${ }^{29}$, A. M. Taylor $^{4}$, R. Terrier $^{29}$,

L. Tibaldo ${ }^{3}$, D. Tiziani ${ }^{34}$, M. Tluczykont ${ }^{2}$, C. Trichard ${ }^{20}$, R. Tuffs $^{3}$, Y. Uchiyama ${ }^{41}$, D. J. van der Walt ${ }^{1}$, C. van Eldik ${ }^{34}$, C. van Rensburg ${ }^{1}$, B. van Soelen ${ }^{38}$, G. Vasileiadis ${ }^{17}$, J. Veh ${ }^{34}$, C. Venter ${ }^{1}$, A. Viana ${ }^{3}$, P. Vincent ${ }^{16}$, J. Vink ${ }^{9}$, F. Voisin ${ }^{14}$, H. J. Völk ${ }^{3}$, T. Vuillaume ${ }^{23}$, Z. Wadiasingh ${ }^{1}$, S. J. Wagner ${ }^{24}$, P. Wagner ${ }^{7}$, R. M. Wagner ${ }^{26}$, R. White ${ }^{3}$, A. Wierzcholska ${ }^{21}$, P. Willmann $^{34}$, A. Wörnlein ${ }^{34}$, D. Wouters ${ }^{18}$, R. Yang $^{3}$, V. Zabalza $^{31}$, D. Zaborov ${ }^{28}$, M. Zacharias ${ }^{24}$, A. A. Zdziarski ${ }^{32}$, A. Zech ${ }^{15}$, F. Zefi ${ }^{28}$, A. Ziegler ${ }^{34}$, and N. Żywucka ${ }^{36}$
\end{abstract}

(Affiliations can be found after the references)

Received 28 July 2016 / Accepted 29 September 2016

\begin{abstract}
Studying the temporal variability of BL Lac objects at the highest energies provides unique insights into the extreme physical processes occurring in relativistic jets and in the vicinity of super-massive black holes. To this end, the long-term variability of the BL Lac object PKS $2155-304$ is analyzed in the high (HE, $100 \mathrm{MeV}<E<300 \mathrm{GeV}$ ) and very high energy (VHE, $E>200 \mathrm{GeV}$ ) $\gamma$-ray domain. Over the course of $\sim 9 \mathrm{yr}$ of H.E.S.S. observations the VHE light curve in the quiescent state is consistent with a log-normal behavior. The VHE variability in this state is well described by flicker noise (power-spectral-density index $\beta_{\mathrm{VHE}}=1.10_{-0.13}^{+0.10}$ ) on timescales larger than one day. An analysis of $\sim 5.5 \mathrm{yr}$ of $\mathrm{HE}$ Fermi-LAT data gives consistent results $\left(\beta_{\mathrm{HE}}=1.20_{-0.23}^{+0.21}\right.$, on timescales larger than 10 days) compatible with the VHE findings. The HE and VHE power spectral densities show a scale invariance across the probed time ranges. A direct linear correlation between the VHE and HE fluxes could neither be excluded nor firmly established. These long-term-variability properties are discussed and compared to the red noise behavior $(\beta \sim 2)$ seen on shorter timescales during VHE-flaring states. The difference in power spectral noise behavior at VHE energies during quiescent and flaring states provides evidence that these states are influenced by different physical processes, while the compatibility of the HE and VHE long-term results is suggestive of a common physical link as it might be introduced by an underlying jet-disk connection.
\end{abstract}

Key words. galaxies: active - BL Lacertae objects: individual: PKS 2155-304 - gamma rays: galaxies - galaxies: jets - galaxies: nuclei radiation mechanisms: non-thermal

\section{Introduction}

One of the most striking properties of BL Lacertae objects is their variability across the electromagnetic spectrum from radio to $\gamma$ rays and accros the temporal spectrum from minutes to

\footnotetext{
^ Corresponding authors: H.E.S.S. Collaboration,

e-mail: contact.hess@hess-experiment.eu

$\dagger$ Deceased.
}

years. In the current standard paradigm of active galactic nuclei (e.g., Urry \& Padovani 1995) the observed nonthermal emission is produced in two-sided collimated, relativistic plasma outflows (jets) closely aligned with the line of sight, so that the intrinsic emission appears enhanced because of Doppler-boosting effects. The jets are powered by a central engine consisting of a supermassive black hole surrounded by an accretion disk. Characterizing the temporal variability provides one of the key diagnostics 
for the physical conditions in these systems, for example the jet-disk connection, location of the emitting region, or dominant emission processes.

The BL Lac PKS 2155-304 (redshift $z=0.116$; Falomo et al. 1993) has been observed with the High Energy Stereoscopic System (H.E.S.S.; Hinton 2004) at very high-energy $\gamma$-rays (VHE; $E>200 \mathrm{GeV}$ ) since 2002 (e.g., Abramowski et al. 2010, and references therein). The source underwent an extreme VHE flux outburst in July/August 2006 with peak fluxes exceeding the average flux level of the longterm emission by a factor of $\sim 100$, during which it showed rapid variability on timescales as short as $3 \mathrm{~min}$ (Aharonian et al. 2007). The stochastic VHE variability during this flaring state has been characterized as power-law noise $\left(\propto f^{-\beta}\right.$, where $f$ is the frequency) with an index $\beta \sim 2$. A detailed analysis of the VHE data from 2005-2007 revealed that the run-by-run light curve of PKS 2155-304 follows a skewed flux distribution, which is well represented by two superposed lognormal distributions (Abramowski et al. 2010, Fig. 3). Excluding the flare data, the flux distribution satisfies a simple lognormal distribution. This provides evidence that the source switches from a quiescent VHE state with minimal activity to a flaring state, and the flux distribution in each state follows a lognormal distribution. Lognormal behavior was first established for accreting Galactic sources such as X-ray binaries by Uttley \& McHardy (2001), linking such a behavior to the underlying accretion process. In a lognormal process, the fluctuations of the flux are on average proportional, or at least correlated, to the flux itself, ruling out additive processes in favor of multiplicative processes. In the case of blazars, a lognormal behavior could thus mark the influence of the accretion disk on the jet (e.g., Giebels \& Degrange 2009; McHardy 2010). Cascade-like events are an example of multiplicative lognormal processes. Density fluctuations in the accretion disk provide one possible realization. If damping is negligible, these fluctuations can propagate inward and couple together to produce a multiplicative behavior. If this is efficiently transmitted to the jet, the $\gamma$-ray emission could be modulated accordingly.

In Sect. 2 we present VHE data from 9 yr of H.E.S.S. observations of PKS 2155-304 in the quiescent state, and partially contemporaneous $\mathrm{HE}$ data from $5.5 \mathrm{yr}$ of observations from the Large Area Telescope (Fermi-LAT), are presented in Sect. 2. In Sect. 3, a detailed time-series analysis is performed. First, the light curves are tested for a lognormal behavior. Then, their variability is characterized as power-law noise with a forward folding method with simulated light curves, taking the sampling of the data, described in Kastendieck et al. (2011) into account. And, finally, the VHE and HE emissions are also analyzed for a possible direct linear correlation. This is the first time that such an extended analysis is performed on the VHE $\gamma$-ray emission of a BL Lac object on timescales as long as nine years. In Sect. 4 the results of the two energy ranges are compared and their implications on the physical properties of PKS 2155-304 are discussed.

\section{Observations and analysis}

H.E.S.S. (VHE): H.E.S.S. is an array of five Imaging Atmospheric Cherenkov Telescopes (IACTs). The first phase of H.E.S.S. began in 2003 with four 12-m telescopes giving an energy threshold $\sim 100 \mathrm{GeV}$. In 2012, a fifth 28 -m telescope was added to the array, reducing the energy threshold to $\sim 50 \mathrm{GeV}$.

The present analysis is based on VHE data taken with the completed H.E.S.S. Phase-I between MJD 53200 (14 July 2004)
Table 1. Values of the log-parabola parameters of the spectrum (Eq. (1)) of PKS 2155-304 used to derive the light curve.

\begin{tabular}{ccc}
\hline \hline Year & $a \pm \sigma_{a}$ & $b \pm \sigma_{b}$ \\
\hline 2004 & $2.95 \pm 0.03$ & $0.37 \pm 0.03$ \\
2005 & $3.27 \pm 0.12$ & $0.25 \pm 0.12$ \\
2006 & $3.27 \pm 0.04$ & $0.24 \pm 0.04$ \\
2007 & $3.38 \pm 0.08$ & $-0.03 \pm 0.07$ \\
2008 & $3.28 \pm 0.04$ & $0.12 \pm 0.03$ \\
2009 & $3.14 \pm 0.08$ & $0.18 \pm 0.07$ \\
2010 & $3.24 \pm 0.08$ & $0.10 \pm 0.07$ \\
2011 & $3.08 \pm 0.10$ & $0.13 \pm 0.08$ \\
2012 & $3.27 \pm 0.01$ & $0.12 \pm 0.09$ \\
\hline
\end{tabular}

and 56246 (15 November 2012), using three or four telescopes. The high flux state from 27 July to 8 August 2006, with an average flux of $(75.2 \pm 0.8) \times 10^{-11} \mathrm{~cm}^{-2} \mathrm{~s}^{-1}$ above $200 \mathrm{GeV}$ is excluded from the data set. The remaining data constitutes the basis for the time-series analysis, and is referred to as the quiescent data set in the following.

In total, about $328 \mathrm{~h}$ of data passed standard quality cuts as defined in Aharonian et al. (2006), with a mean zenith angle of $21^{\circ}$ resulting in an average energy threshold of $178 \mathrm{GeV}$. The data set has been analyzed with the Model analysis chain using standard cuts (de Naurois \& Rolland 2009) above $200 \mathrm{GeV}$.

The total detection significance in the quiescent data set corresponds to $341 \sigma$. The light curve of nightly fluxes is calculated assuming a log-parabolic energy spectrum,

$\mathrm{d} N / \mathrm{d} E \propto E^{-a-b \log E}$,

with $a$ and $b$ corresponding to the best-fit power-law index and curvature index, respectively. In order to take indications of a spectral variability in the VHE domain during the quiescent state (Abramowski et al. 2010) into account, the nightly fluxes are derived with a separate log-parabola fit of the spectrum for each year.

The values for $a$ and $b$ are summarized in Table 1 ; the average values are $\bar{a}=3.209$ and $\bar{b}=0.164$. The unbiased sample variance for the first parameter

$s_{a}^{2}=\frac{1}{n-1} \sum_{i=1}^{n}\left(a_{i}-\bar{a}\right)^{2}=0.017$

is larger than the expected variance $\overline{\sigma_{a}^{2}}=0.005$ because of the uncertainties $\sigma_{a}$ on the individual best-fit values. The same is true for the second parameter with $s_{b}^{2}=0.114$ and $\overline{\sigma_{b}^{2}}=0.005$. This could be indicative of a variable spectrum.

Variations within a season, however, are unlikely to affect the analysis presented here as the inferred small changes in the spectral parameters only result in small changes in the integral flux.

The resulting quiescent light curve has an average flux of $(5.10 \pm 0.41) \times 10^{-11} \mathrm{~cm}^{-2} \mathrm{~s}^{-1}$ above $200 \mathrm{GeV}$ and is shown in Fig. 1. It is characterized by a fractional root mean square (rms) variability (Vaughan et al. 2003)

$F_{\text {var }}=\frac{\sqrt{S_{\Phi}^{2}-\overline{\sigma_{\text {err }}^{2}}}}{\bar{\Phi}}=0.66 \pm 0.01$,

where $\bar{\Phi}$ is the mean flux, $S_{\Phi}^{2}$ is the variance of the fluxes and $\overline{\sigma_{\text {err }}^{2}}$ is the contribution to the variance caused by the measurement 
The H.E.S.S. Collaboration: $\gamma$-ray long-term variability of PKS 2155-304

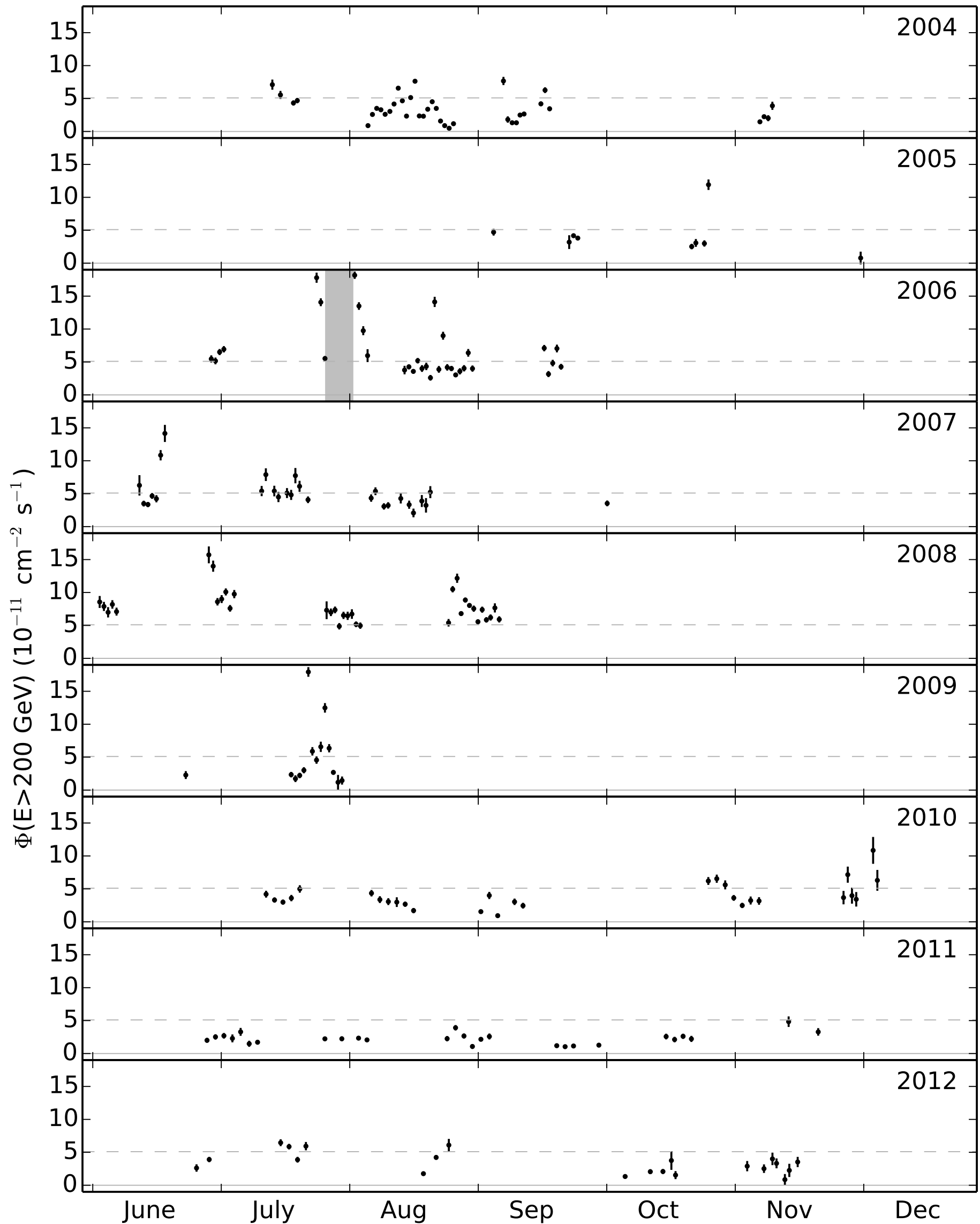

Fig. 1. H.E.S.S. light curve of nightly fluxes above $200 \mathrm{GeV}$ excluding the high state in July/August 2006 (gray shaded area). The gray dashed horizontal line indicates the average flux of the quiescent state. 


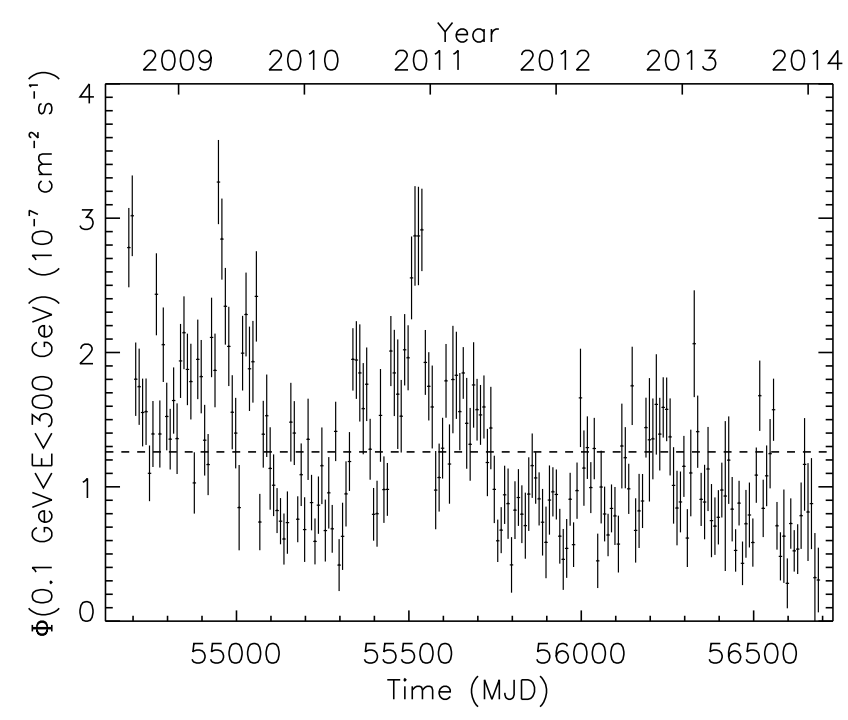

Fig. 2. Light curve of the integral fluxes between 0.1 and $300 \mathrm{GeV}$ in bins of ten days measured with Fermi-LAT. The dashed line indicates the average flux.

errors. The analysis results were cross-checked using different analysis methods and calibration chains (e.g., Aharonian et al. 2006), yielding compatible results.

Fermi-LAT (HE): The Fermi-LAT (Atwood et al. 2009) on board the Fermi satellite is a pair-conversion telescope designed to detect $\mathrm{HE} \gamma$-rays in the energy range from below $20 \mathrm{MeV}$ to more than $300 \mathrm{GeV}$.

The set of observational data for PKS 2155-304 used here covers about $5.5 \mathrm{yr}$, from MJD 54688 (10 August 2008) to 56688 (31 January 2014). Events are selected between $100 \mathrm{MeV}$ and $300 \mathrm{GeV}$ in a region of interest (ROI) of $15^{\circ}$ around PKS 2155-304. The detector is described by the P7REP_SOURCE_V15 instrument response function ${ }^{1}$. The HE light curve is obtained with the tool Enrico (Sanchez \& Deil 2013) using the Fermi Science Tools v9r32p5 $5^{2}$, with a 10 -day binning to ensure enough statistics in each bin. The prefactor of the diffuse galactic background (gll_iem_v05) and the normalization of the isotropic diffuse emission (iso_source_v05) are left free to vary in the likelihood fit.

All sources from the third LAT source catalog (3FGL; Acero et al. 2015) within $15^{\circ}$ of PKS 2155-304 are included in the model to ensure a good background modeling. The HE spectra of PKS 2155-304 and all sources within $3^{\circ}$ are fitted following the spectral shape of the 3FGL catalog. The spectra of PKS 2155-304, 3FGL J2151.6-2744 and 3FGL J2159.2-2841 are modeled with a simple power law, while for 3FGL J2151.83025 a log-parabolic shape is assumed. The indices and prefactors are left free. All other components are fixed to the values in the 3FGL catalog. PKS 2155-304 is detected with a significance of $156 \sigma$ with a spectral index of $1.83 \pm 0.01$. The photon counts of PKS 2155-304 are integrated into 201 bins of ten days. The resulting light curve has an average flux of $1.20 \pm 0.03 \times 10^{-7} \mathrm{~cm}^{-2} \mathrm{~s}^{-1}$ between $100 \mathrm{MeV}$ and $300 \mathrm{GeV}$ with a $F_{\text {var }}=0.41 \pm 0.02$ (Eq. (3)) and is shown in Fig. 2.

\footnotetext{
1 http://fermi.gsfc.nasa.gov/ssc/data/analysis/ documentation/Pass7REP_usage.html

2 Cf. the Fermi Science Support Center web site http://fermi. gsfc.nasa.gov/ssc/
}

Table 2. Values of the reduced $\chi^{2}$ and associated probability for the Gaussian fits of the flux and log flux distributions for each light curve.

\begin{tabular}{lccccc}
\hline & \multicolumn{2}{c}{$\Phi$} & \multicolumn{3}{c}{$\log \Phi$} \\
& $\chi^{2} /$ d.o.f. & Prob & $\chi^{2} /$ d.o.f. & Prob & $\sigma$ \\
\hline H.E.S.S. & $50.8 / 17$ & $10^{-5}$ & $11.9 / 13$ & 0.54 & 5.39 \\
Fermi & $21.6 / 12$ & 0.04 & $15.0 / 11$ & 0.18 & 2.57 \\
\hline
\end{tabular}

Notes. The parameter $\sigma$ is the significance level on which a lognormal distribution is preferred to a normal distribution.

Table 3. Values of the reduced $\chi^{2}$ of the constant and linear fits of the scatter plots shown in Fig. 3 for each light curve with values for the significance $\sigma$, correlation factor $\rho$, and Kendall rank $\tau$.

\begin{tabular}{lccc}
\hline \hline & Constant & Linear increase \\
& $\chi^{2} /$ d.o.f. & $\chi^{2} /$ d.o.f. & $\sigma$ \\
\hline H.E.S.S. & 5.78 & 0.89 & 6.33 \\
Fermi & 0.936 & 0.107 & 2.75 \\
\hline \multicolumn{4}{c}{$\tau$} \\
\hline H.E.S.S. & $0.86 \pm 0.11$ & $0.78 \pm 0.26$ \\
Fermi & $0.93 \pm 0.19$ & $0.69 \pm 0.25$ \\
\hline
\end{tabular}

\section{Time-series analysis}

To characterize the long-term variability of PKS 2155-304, the $\mathrm{HE}$ and VHE data sets are analyzed with respect to lognormality and power-law noise.

Lognormality: A possible lognormal behavior of PKS 2155-304 is investigated by examining the distribution of the fluxes and studying the correlation between the flux levels and the intrinsic variability. The flux and log-flux distributions of each light curve are fitted by a Gaussian using a $\chi^{2}$ fit. The goodness of the fits are summarized in Table 2. In both cases, a Gaussian fits the log-flux distribution better than the flux distribution, with a significance level of $\sigma>5$ for the VHE and $\sigma>2$ for the HE data, respectively. For the VHE data the probability representing the goodness of fit is about $10^{4}$ times higher for the log-flux distribution when compared to the flux distribution. For the HE data this ratio is on the order of 10. Thus, while the HE $\gamma$-ray flux of PKS 2155-304 in this approach shows only an indication, the VHE $\gamma$-ray flux data provide evidence for a lognormal behavior.

In addition, the variability-flux relation, estimated by the excess rms (Eq. (4)), is investigated for a possible correlation (Uttley et al. 2005). The excess rms estimates the intrinsic variability of a time series by subtracting the contribution of the measurement errors. It is defined as

$\sigma_{\mathrm{XS}}=\sqrt{S^{2}-\overline{\sigma_{\mathrm{err}}^{2}}}$

where $S^{2}$ is the variance and $\overline{\sigma_{\text {err }}^{2}}$ the mean square of the statistical error of the data (Vaughan et al. 2003). Here $\sigma_{\mathrm{XS}}$ is calculated for bins of the light curves each containing at least 20 light curve points. They are plotted versus the average fluxes $\bar{\Phi}$ of the corresponding bins for both light curves in Fig. 3.

To test the possible correlation between the flux and its variability, the scatter plots are fitted by a constant as well as a linear ascending slope. The fit results are summarized in Table 3. The results reveal a preference greater than $6 \sigma$ for the linear fit to the VHE data while HE data only show an indication of linearity. To characterize this behavior beyond the fit of a linear correlation, the nonparametric correlation factor Spearman $\rho$ and 

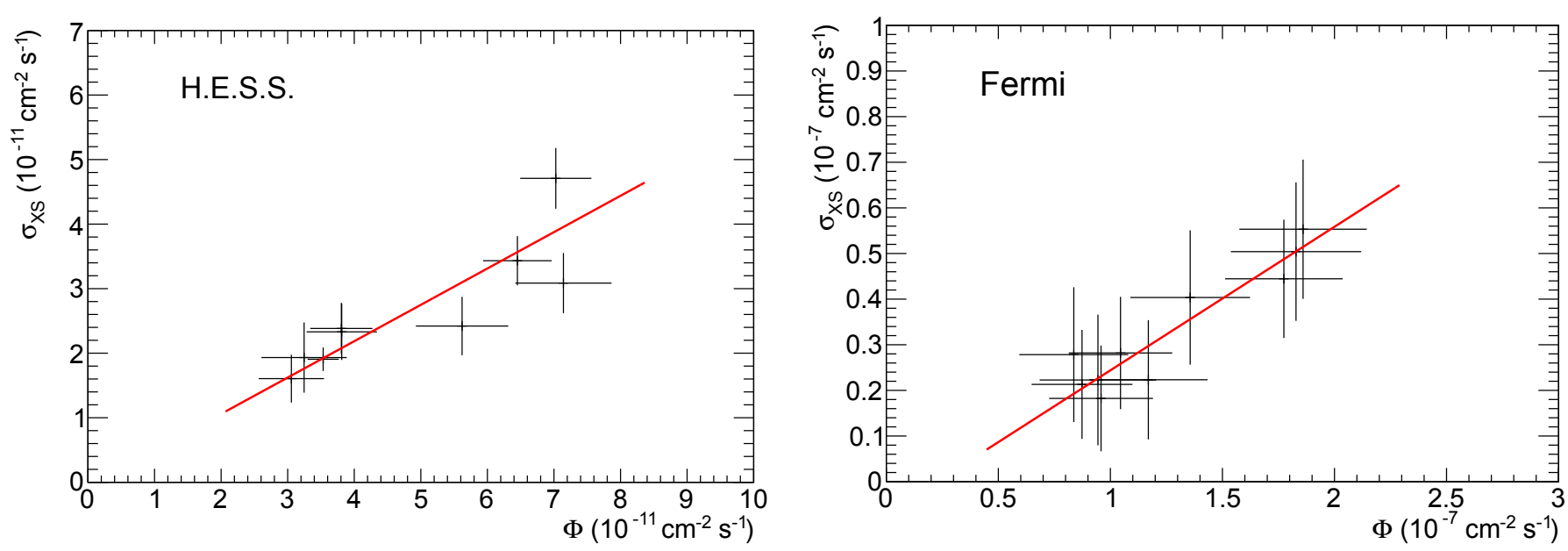

Fig. 3. Scatter plot of the excess rms and the average flux for the H.E.S.S. (left) and Fermi-LAT (right) data. Each flux and excess rms values are computed using at least 20 adjacent light curve points. A linear fit is shown in red.

the Kendall rank $\tau$, which measure the ordering of the points, (Gleissner et al. 2004) are calculated. In all cases $\rho>0.85$ and $\tau>0.65$, meaning that $\sigma_{\mathrm{XS}}$ and $\bar{\Phi}$ show a strong correlation. This implies that the fluctuations of the flux are in fact correlated with the flux.

The preceding analysis shows that the HE and VHE flux distributions of PKS 2155-304 during the quiescent state are compatible with lognormal distributions. When the two energy bands are compared, the lognormal behavior is much more evident in the VHE data set. In addition, for both energy bands the variability amplitude of the flux is correlated with the flux level, supporting the conclusion that lognormality is an intrinsic characteristic of the long-term $\gamma$-ray emission in PKS 2155-304. A similar result has also been reported for the VHE flaring state of PKS 2155-304 in 2006 (Abramowski et al. 2010).

Power-law noise: the flux variability of active galaxies has frequently been characterized as power-law noise (e.g., Lawrence \& Papadakis 1993). The power spectral density (PSD), i.e., the square modulus of the discrete Fourier transform (Priestley 1967), of such a light curve follows a power law $\propto f^{-\beta}$, where $f$ is the temporal frequency and usually $1 \lesssim \beta \lesssim 2$. The PSD reveals how the variability amplitude is distributed among the timescales. In a power-law noise light curve $(\beta>0)$ the flux variations on longer timescales dominate the variations on shorter timescales. The total variance of such a light curve tends to grow with its length. For $\beta=0$ the variability power is equal on each time cale, resulting in white noise, where the fluxes at all times are uncorrelated.

To study the variability characteristics, we first hypothesize that the light curves can be described by power-law noise with a lognormal behavior with $\beta \geq 0$ as the only free parameter. Three different methods are then used to characterize the variability of the light curves: the Lomb-Scargle Periodogram (LSP), which is a method to approximate the PSD (Lomb 1976; Scargle 1982); the first-order Structure Function (SF; Simonetti et al. 1985) and the Multiple Fragments Variance Function (MFVF; Kastendieck et al. 2011), which are both representations of the PSD in the time domain.

A forward-folding method with sets of $10^{4}$ simulated light curves for each value of $\beta$ and a maximum likelihood estimator are applied to estimate the best-fit parameter $\beta$, as described in Kastendieck et al. (2011). The light curves are simulated with a lognormal behavior, so that the logarithm of the intrinsic light curve $\Phi_{\text {intr }}$ is power-law noise with a Gaussian behavior of mean $\mu$ and standard deviation $\sigma$.

$\log \left(\Phi_{\text {intr }}\right) \mapsto \mathcal{N}(\mu, \sigma)$

The PSD of the simulated power-law noise is generated on a frequency range $\left[(10 T)^{-1}, 5 \mathrm{~d}^{-1}\right]$ where $T_{\text {H.E.S.S. }}=3047 \mathrm{~d}$ and $T_{\text {Fermi }}=2000 \mathrm{~d}$ are the lengths of the measured light curves, respectively. The power-law noise is rebinned to ten days for the Fermi-LAT analysis corresponding to the binning of the PKS 2155-304 lightcurve. The light curves are then downsampled to the real observation times, the fluxes are rescaled to the average and variance of the measured fluxes, and measurement errors are simulated. The parameter space is sampled with $\beta=$ $0.0,0.1, \ldots, 3.0$. The SFs, LSPs and MFVFs are equally binned in $\log _{10}$ scale with 50, 20, and 5 bins per decade, respectively.

Owing to the binning, the smallest resolvable timescale in the Fermi-LAT light curve is $\tau_{0}=10 \mathrm{~d}$ and the corresponding frequency is $f_{\text {high }}=\left(2 \tau_{0}\right)^{-1}=(20 \mathrm{~d})^{-1}$. For the H.E.S.S. light curve the smallest resolvable timescale is $\tau_{0}=1 \mathrm{~d}$ corresponding to $f_{\text {high }}=(2 \mathrm{~d})^{-1}$. The LSPs are characterized on a frequency range $\left[10^{-4} \mathrm{~d}^{-1}, f_{\text {high }}\right]$, while the lowest timescale for the SFs and MFVFs is $\tau_{0}$. The methods are also sensitive to variations that occur on timescales larger than the lengths of the light curves and that appear as long-term trends. It is therefore also useful to calculate the LSP on frequencies $<T^{-1}$ to reveal such information.

The application of these methods to the H.E.S.S. light curve in the quiescent state gives best-fit parameters

$\beta_{\mathrm{LSP}}=1.10_{-0.16}^{+0.14}, \quad \beta_{\mathrm{SF}}=1.00_{-0.15}^{+0.11}, \quad \beta_{\mathrm{MFVF}}=1.10_{-0.13}^{+0.10}$.

A goodness of fit test is applied where the maximum likelihood values of simulated LSPs, SFs and MFVFs, respectively, are used as test statistics. For this, new simulated sets of $10^{4}$ light curves of the best-fit parameters are analyzed with the likelihood estimator. The distribution of their maximum likelihood values is compared with the respective value of PKS 2155-304. Assuming the model to be correct, the values should be compatible. The quantile that has a smaller likelihood than the PKS 2155-304 data is used to estimate the $p$-value $p$. The smallest value of $p=11 \%$ is found with the MFVF. The hypothesis that the data can be described by a single power-law noise model is thus not rejected. 
The MFVF gives the most precise value, which is taken here as the final value:

$\beta_{\mathrm{VHE}}=1.10_{-0.13}^{+0.10}$.

The best-fit values of the other methods are compatible within the uncertainties.

The LSP, SF and MFVF of the measured light curves together with the probability density functions (PDF) of the simple power-law noise model that best fits the data are shown in Fig. A.1. The probability is normalized to unity in each frequency bin. The uncertainties of $\beta$ are found with the distributions illustrated in Fig. A.2.

For comparison, as a second hypothesis, an extended model is investigated: a maximum timescale is considered, above which the variance does not increase any further. The existence of such a timescale is expected, as otherwise the variance of the light curve and therefore the flux would increase to infinity with time. This is represented by a break in the PSD to a constant level $(\beta=0)$ at the corresponding frequency $f_{\min }$, which is treated as an additional free parameter, with a sampling $\log _{10}\left(f_{\min } / \mathrm{d}^{-1}\right)=$ $-4.4,-4.2, \ldots,-1.0$.

The likelihood estimators with the three methods give bestfit values or $1 \sigma$ upper limits,

$\beta_{\mathrm{LSP}}=1.10_{-0.09}^{+0.28}$,

$\log _{10}\left(f_{\min , \mathrm{LSP}} / \mathrm{d}^{-1}\right)=-3.80_{-0.31}^{+1.12}<-2.68$,

$\beta_{\mathrm{SF}}=1.00_{-0.07}^{+0.24}$

$\log _{10}\left(f_{\min , \mathrm{SF}} / \mathrm{d}^{-1}\right)=-3.80_{-0.18}^{+1.61}<-2.19$,

$\beta_{\mathrm{MFVF}}=1.10_{-0.06}^{+0.23}$

$\log _{10}\left(f_{\min , \mathrm{MFVF}} / \mathrm{d}^{-1}\right)=-3.60_{-0.34}^{+1.41}$.

The best-fit values of $\log _{10}\left(f_{\min } / \mathrm{d}^{-1}\right)=-3.80$ for the LSP and SF is near the formal limit of our analysis, which is constrained to -4.4 by the lengths of the simulated light curves. The results together with the $1 \sigma$ uncertainties to higher values $(+1.12$ and $+1.61)$ are therefore treated as $1 \sigma$ upper limits. The best likelihood for the MFVF is given for a break in the PSD. However, the goodness-of-fit $\mathrm{p}$ value of $10 \%$ is not improved compared to $11 \%$ for the first hypothesis (assuming no break). This and the LSP and MFVF findings thus do not reveal any preference for the existence of such a break in the sampled frequency range.

An analogous analysis is performed on the Fermi-LAT light curve. The following best-fit parameters for the simple powerlaw model are compatible:

$\beta_{\mathrm{LSP}}=1.10_{-0.31}^{+0.26}, \quad \beta_{\mathrm{SF}}=1.20_{-0.31}^{+0.22}, \quad \beta_{\mathrm{MFVF}}=1.20_{-0.23}^{+0.21}$.

The most constraining result, again given by the MFVF, is taken as the final value:

$\beta_{\mathrm{HE}}=1.20_{-0.23}^{+0.21}$.

The goodness-of-fit tests yield $p$ values $>6 \%$. Inspection of the LSP plot in Fig. A.1 for the Fermi-LAT data reveals a peak at $\sim(670-700)$ days, which is indicative of a possible HE periodicity on the noted timescale and influencing the $p$ value for this analysis. It is interesting to note that a tentative $\mathrm{HE}$ periodicity of $\sim(620-660)$ days has already been reported (Sandrinelli et al. 2014).
A comparative analysis of the extended model (power law with a break) gives compatible results with

$\beta_{\mathrm{LSP}}=1.10_{-0.11}^{+0.46}$,

$\log _{10}\left(f_{\min , \mathrm{LSP}} / \mathrm{d}^{-1}\right)=-4.20_{-0.12}^{+1.42}<-2.78$,

$\beta_{\mathrm{SF}}=1.20_{-0.11}^{+0.44}$,

$\log _{10}\left(f_{\min , \mathrm{SF}} / \mathrm{d}^{-1}\right)=-4.20_{-0.12}^{+1.40}<-2.80$,

$\beta_{\mathrm{MFVF}}=1.30_{-0.08}^{+0.54}$

$\log _{10}\left(f_{\min , \mathrm{MFVF}} / \mathrm{d}^{-1}\right)=-3.40_{-0.12}^{+0.74}$.

The best-fit values for the LSP and the SF are both $\log _{10}\left(f_{\min } / \mathrm{d}^{-1}\right)=-4.20$. Including the $1 \sigma$ uncertainties to higher values $(+1.42$ and +1.40$)$ they are treated as $1 \sigma$ upper limits.

The goodness-of-fits do not improve. The results therefore give no indication for the presence of a break frequency in the PSD in the sampled frequency range. See Table 4 for a summary of these and related results.

Correlations: The VHE and HE light curves are furthermore analyzed for a possible direct correlation with the discrete correlation function (DCF; Edelson \& Krolik 1988) using a binning of $30 \mathrm{~d}$. The results are compared with the DCFs of simulated H.E.S.S. and Fermi-LAT light curves following one of the two hypotheses: (1) the H.E.S.S. and the Fermi-LAT light curves are characterized by flicker noise $(\beta=1.1)$ without any correlation; (2) the fluxes of such light curves obey a perfect, direct linear correlation.

For each hypothesis $10^{4}$ pairs of corresponding light curves are simulated and their DCFs are calculated. These DCFs are then combined in a two-dimensional histogram which is treated as a PDF. The hypotheses are tested according to the goodnessof-fit test described above: for the measured DCF the likelihood is calculated with the PDF. Also for the simulated DCFs the likelihoods are calculated and are used as a test statistic for calculating the $p$ value. Both hypotheses are compatible with the measured data with $p$ values for (1) of $41 \%$, and for (2) of $59 \%$, respectively. Accordingly, these results neither give a clear preference nor do they allow the rejection of a direct correlation.

\section{Discussion and conclusions}

For the first time the temporal variability of the VHE emission of PKS 2155-304 in the quiescent state has been analyzed on timescales from days up to more than nine years. The variability of the long-term quiescent VHE light curve as measured with H.E.S.S. provides evidence for a lognormal behavior and is compatible with power-law noise process on timescales $>1 \mathrm{~d}$. The VHE PSD on these timescales is consistent with a power law $\left(\propto f^{-\beta}\right)$ with an index of $\beta_{\mathrm{VHE}}=1.10_{-0.13}^{+0.10}$ (flicker noise). On the other hand, the PSD for the H.E.S.S. data during the flaring period in 2006 is consistent with a power law of slope $\beta=2.06 \pm 0.21$ (red noise) on timescales $<3 \mathrm{~h}$ with indications for a possible break in the SF between 3 and $20 \mathrm{~h}$ (Abramowski et al. 2010). In the context of accretion-powered sources, X-ray variability with similar characteristics (powerlaw noise with $\beta \sim 1-2$, and lognormal behavior) has often been related to random fluctuations of the disk parameter $\alpha$ on local viscous timescales (e.g., Lyubarskii 1997; King et al. 2004). The 
The H.E.S.S. Collaboration: $\gamma$-ray long-term variability of PKS 2155-304

Table 4. Characteristics of the power spectral densities of PKS 2155-304 at different energies and timescales.

\begin{tabular}{|c|c|c|c|c|c|c|c|}
\hline $\begin{array}{l}\text { Energy } \\
\text { range }\end{array}$ & Instrument & $\begin{array}{c}\text { Range of } \\
\log _{10}\left(f / \mathrm{d}^{-1}\right)\end{array}$ & Method & $\beta$ & $\log _{10}\left(f_{\min } / \mathrm{d}^{-1}\right)$ & $\begin{array}{c}\text { Goodness } \\
\text { of fit }\end{array}$ & Ref. \\
\hline$>200 \mathrm{GeV}$ & H.E.S.S. & {$[-4.0,-0.3]$} & LSP, FF & $1.10^{+0.14}$ & no break (fixed) & $56 \%$ & \\
\hline$>200 \mathrm{GeV}$ & H.E.S.S. & {$[-3.5,0.0]$} & $\mathrm{SF}, \mathrm{FF}$ & $1.00_{-0.15}^{+0.11}$ & no break (fixed) & $48 \%$ & \\
\hline$>200 \mathrm{GeV}$ & H.E.S.S. & {$[-3.5,0.0]$} & MFVF, FF & $1.10_{-0.13}^{+0.10}$ & no break (fixed) & $11 \%$ & \\
\hline$>200 \mathrm{GeV}$ & H.E.S.S. & {$[-4.0,-0.3]$} & LSP, FF & $1.10_{-0.09}^{+0.28}$ & $-3.80^{+1.12}<-2.68$ & $51 \%$ & \\
\hline$>200 \mathrm{GeV}$ & H.E.S.S. & {$[-3.5,0.0]$} & $\mathrm{SF}, \mathrm{FF}$ & $1.00_{-0.07}^{+0.09}$ & $-3.80_{-0.18}^{+1.61}<-2.19$ & $43 \%$ & \\
\hline$>200 \mathrm{GeV}$ & H.E.S.S. & {$[-3.5,0.0]$} & MFVF, FF & $1.10_{-0.06}^{+0.03}$ & $-3.60_{-0.14}^{+1.41}$ & $10 \%$ & \\
\hline $0.1-300 \mathrm{GeV}$ & Fermi-LAT & {$[-4.0,-1.3]$} & LSP, FF & $1.10_{-0.031}^{+0.26}$ & no break (fixed) & $6.3 \%$ & \\
\hline $0.1-300 \mathrm{GeV}$ & Fermi-LAT & {$[-3.3,-1.0]$} & $\mathrm{SF}, \mathrm{FF}$ & $1.20_{-0.31}^{+0.22}$ & no break (fixed) & $46 \%$ & \\
\hline $0.1-300 \mathrm{GeV}$ & Fermi-LAT & {$[-3.3,-1.0]$} & MFVF, FF & $1.20_{-0.23}^{+0.21}$ & no break (fixed) & $40 \%$ & \\
\hline $0.1-300 \mathrm{GeV}$ & Fermi-LAT & {$[-4.0,-1.3]$} & LSP, FF & $1.10_{-0.11}^{+0.46}$ & $-4.20_{-012}^{+1.42}<-2.78$ & $2.4 \%$ & \\
\hline $0.1-300 \mathrm{GeV}$ & Fermi-LAT & {$[-3.3,-1.0]$} & SF, FF & $1.20_{-0.11}^{+0.44}$ & $-4.20_{-0.12}^{+1.40}<-2.80$ & $33 \%$ & \\
\hline $0.1-300 \mathrm{GeV}$ & Fermi-LAT & {$[-3.3,-1.0]$} & MFVF, FF & $1.30_{-0.08}^{+0.54}$ & $-3.40_{-0.12}^{+0.74}$ & $30 \%$ & \\
\hline$>200 \mathrm{GeV}$ & H.E.S.S. ${ }^{a}$ & {$[0.9,2.6]$} & $\mathrm{SF}, \mathrm{FF}$ & $2.06 \pm 0.21$ & {$[0.1,0.9]$} & - & (1) \\
\hline $0.1-300 \mathrm{GeV}$ & Fermi-LAT ${ }^{b}$ & {$[-2.0,-0.9]$} & PSD, fit & $0.577 \pm 0.332$ & - & - & (2) \\
\hline $0.1-300 \mathrm{GeV}$ & Fermi-LAT ${ }^{c}$ & {$[-3.2,-0.7]$} & PSD, fit & $0.64_{-0.50}^{+0.79}$ & $<-1.7$ & - & (3) \\
\hline $2.5-20 \mathrm{keV}$ & $\mathrm{RXTE}^{d}$ & {$[-0.9,0.0]$} & PSD, fit & $1.46 \pm 0.10$ & - & - & (4) \\
\hline $2.5-20 \mathrm{keV}$ & $\mathrm{RXTE}^{d}$ & {$[0.0,1.9]$} & PSD, fit & $2.23 \pm 0.10$ & - & - & (4) \\
\hline Optical & Geneva & {$[-1.2,2.0]$} & SF, fit & $2.4_{-0.2}^{+0.3}$ & - & - & (5) \\
\hline Optical & ROTSE & {$[-4.4,-1.3]$} & MFVF, FF & $1.8_{-02}^{+0.1}$ & $-3.0_{-0.4}^{+0.3}$ & - & (6) \\
\hline Optical & SMARTS & {$[-2.4,-0.9]$} & PSD, fit & $2.2_{-0.4}^{+0.2}$ & - & - & (7) \\
\hline
\end{tabular}

Notes. Range of $\log _{10}\left(f / \mathrm{d}^{-1}\right)$ : the range over which the PSD is characterized. Method: the methods used for the analysis; forward folding method (FF), best fit of the slope by, e.g. a $\chi^{2}$-test (fit), Structure Function (SF), Lomb-Scargle Periodogram (LSP) and Multiple Fragments Variance Function (MFVF). Goodness of fit: the $p$-value obtained with simulated light curves. Upper part - results from this work. Lower part - results reported in the literature with superscripts referring to: ${ }^{(a)}$ H.E.S.S. flaring state: a break was detected with a $95 \%$ confidence in the SF. It shall be noted that a break in the SF is at a $\sim 3$ times larger frequency than in the intrinsic PSD. ${ }^{(b)}$ Based on aperture photometric Fermi-LAT light curves provided by the Fermi Science Support Center at http://fermi .gsfc.nasa.gov/ssc/data/access/lat/msl_lc/. ${ }^{(c)}$ Model results assuming a superposition of Ornstein-Uhlenbeck processes (OU) and using $4 \mathrm{yr}$ of Fermi-LAT data. A slight preference for a single OU with different slope is reported. ${ }^{(d)}$ Based on non-simultaneous data.

References. (1) Abramowski et al. (2010); (2) Nakagawa \& Mori (2013); (3) Sobolewska et al. (2014); (4) Kataoka et al. (2001); (5) Paltani et al. (1997); (6) Kastendieck et al. (2011); (7) Chatterjee et al. (2012).

current VHE findings can in principle be interpreted in two different ways:

(1) The PSD slope of the VHE variability is stationary. It follows a power law with a transition (break) from $\beta \sim 2$ to $\sim 1$ somewhere between 0.1 and $\sim 1 \mathrm{~d}$. This can be compared to the X-ray PSDs of Seyfert AGN and radio galaxies. The PSD of Seyfert AGN exhibit a power-law behavior $\beta \simeq 1$ at low frequency, steepening to $\beta \gtrsim 2$ on timescales shorter than some break time $T_{\text {br }}$ (e.g., McHardy et al. 2006). Two radio galaxies, 3C 111 and 3C 120 show a similar behavior in $\mathrm{X}$-ray with a power-law slope of $\sim 2$ for 3C 111 (Chatterjee et al. 2011) and a steepening of the PSD at high frequency for 3C 120 (Chatterjee et al. 2009). Interestingly, for PKS 2155-304 a break time $T_{\mathrm{br}} \sim 1 \mathrm{~d}$ has been suggested earlier (Kataoka et al. 2001, cf. also Table 1), based on (nonsimultaneous) X-ray data (cf. also Emmanoulopoulos et al. 2010, for caveats). In the case of Seyfert AGN a simple quantitative relationship between $T_{\mathrm{br}}$, the observed (bolometric) luminosity $L_{\mathrm{B}}$ and the black hole mass $M_{\mathrm{BH}}$ has been found (McHardy et al. 2006). Although PKS 2155-304 is not a radio-quiet object, a similar relation could apply if its characteristic timing properties are caused by an external process (e.g., originate in the accretion flow, see for instance; McHardy 2010). In terms of the accretion rate $\dot{m}_{\mathrm{E}}$ (expressed in units of the Eddington rate), the relevant scaling relation for a standard disk configuration becomes $\left(T_{\mathrm{br}} / 1 \mathrm{~d}\right) \simeq 0.7\left(M_{\mathrm{BH}} / 10^{8} M_{\odot}\right)^{1.12} / \dot{m}_{\mathrm{E}}^{0.98}$. If this would apply to PKS 2155-304, then relatively high accretion rates ( 20.1 Eddington rate for $M_{\mathrm{BH}} \geq 10^{8} M_{\odot}$ ) would be implied even for the quiescent VHE state. This may suggest that in this context the break time is more likely related to a change in accretion flow conditions such as a transition from an advection-dominated to a standard disk configuration.

(2) Alternatively, the power-law indices of the PSD could be different during the quiescent and flaring states, as they are possibly related to different physical processes and/or spatial locations. The extreme characteristics of the flaring state (including apparent lognormality and a red-noise behavior down to minutes) seem to require rather exceptional conditions to account for it (e.g., Rieger \& Volpe 2010; Biteau \& Giebels 2012; Narayan \& Piran 2012). This could support a variability origin differing from the origin in the quiescent state. Further limits on the possible cross-over timescale will be important to distinguish between these two scenarios.

The HE light curve, as measured with Fermi-LAT, is found to be compatible with a power-law noise of slope $\beta_{\mathrm{HE}}=1.20_{-0.23}^{+0.21}$ on timescales larger than ten days. This value differs slightly from the earlier reported best-fit $\beta=1.7 \pm 0.3$ for the average PSD of the six brightest Fermi-LAT BL Lacs (including PKS 2155-304) 
based on the first 11 months of data (Abdo et al. 2010). It is compatible with more recent indications for a flatter slope $(\beta \sim 1)$ for high-frequency-peaked BL Lac (HBL) objects (S. Larsson, priv. comm.). The HE slope is also compatible with the VHE results for the quiescent data set, suggesting that the PSDs on these timescales are shaped by similar processes. Owing to instrumental noise and observational gaps a possible direct correlation between the VHE and the HE light curves could neither be excluded nor firmly established.

The HE and VHE PSDs show a scale invariance on timescales from weeks up to at least the $1 \sigma$-lower limits $\gtrsim 600 \mathrm{~d}$ and $\gtrsim 200 \mathrm{~d}$, respectively. A maximum timescale of $10^{3}$ days has been reported in the optical range (see Table 1). If the maximum timescale was related to the radial infall time in the accretion disk, then a possible outer radius of $r_{\mathrm{d}} \simeq\left(\alpha \sqrt{G M_{\mathrm{BH}}} f_{\text {min }}^{-1}\right)^{2 / 3} \gtrsim$ $4 \times 10^{16}(\alpha / 0.3)^{2 / 3}\left(t_{\max } / 1000 \mathrm{~d}\right)^{2 / 3}\left(M_{\mathrm{BH}} / 10^{8} M_{\odot}\right)^{1 / 3} \mathrm{~cm}$ might be inferred for an advection-dominated system (Lyubarskii 1997). However a significant detection of such a maximum timescale in the $\gamma$-ray range will probably require longer light curves that exceed this timescale by an order of magnitude.

For both data sets of PKS 2155-304, the flux distributions during the quiescent state are compatible with lognormal distributions, where the result is more significant for the VHE data than for the HE data. A hint of lognormal behavior was found in Abramowski et al. (2010) where the distribution of the fluxes of the quiescent state of 2005-2007 were following a lognormal distribution. This suggests that multiplicative, i.e., self-boosting processes dominate the variability. It is interesting to note that in the context of galactic X-ray binaries, where lognormal flux variability has first been established, such a behavior is thought to be linked to the underlying accretion process (Uttley \& McHardy 2001). In the AGN context, evidence for lognormality on different timescales has in the meantime also been found in several sources, for example, in the X-ray band for the BL Lac object BL Lacertae (Giebels \& Degrange 2009), in the TeV band for the BL Lac object Markarian 501 (Tluczykont et al. 2010; Chakraborty et al. 2015), and in the X-ray band for the Seyfert 1 galaxy IRAS 13 244-3809 (Gaskell 2004).

Further observations with H.E.S.S. II and the planned Cherenkov Telescope Array will allow us to improve the characterization of the PSD at high frequencies due to their better sensitivities (Sol et al. 2013). The larger energy range will make it possible to close the gap to the Fermi band, helping to improve our understanding of the degree of convergence (e.g., possible correlations and similar processes) between the HE and the VHE domain. A clear characterization of the $\gamma$-ray variability of different sources similar to PKS 2155-304 will also be important to improve our understanding of the physical processes separating different source classes.

Acknowledgements. The support of the Namibian authorities and of the University of Namibia in facilitating the construction and operation of H.E.S.S. is gratefully acknowledged, as is the support by the German Ministry for Education and Research (BMBF), the Max Planck Society, the German Research Foundation (DFG), the French Ministry for Research, the CNRS-IN2P3 and the Astroparticle Interdisciplinary Programme of the CNRS, the UK Science and Technology Facilities Council (STFC), the IPNP of the Charles University, the Czech Science Foundation, the Polish Ministry of Science and Higher Education, the South African Department of Science and Technology and Nationa Research Foundation, the University of Namibia, the Innsbruck University, the Austrian Science Fund (FWF), and the Austrian Federal Ministry for Science Research and Economy, and by the University of Adelaide and the Australian Research Council. We appreciate the excellent work of the technical support staff in Berlin, Durham, Hamburg, Heidelberg, Palaiseau, Paris, Saclay, and in Namibia in the construction and operation of the equipment. This work benefited from services provided by the H.E.S.S. Virtual Organisation, supported by the national resource providers of the EGI Federation.

\section{References}

Abdo, A. A., Ackermann, M., Ajello, M., et al. 2010, ApJ, 722, 520 Abramowski, A., Acero, F., Aharonian, F., et al. 2010, A\&A, 520, A83 Acero, F., Ackermann, M., Ajello, M., et al. 2015, ApJS, 218, 23

Aharonian, F., Akhperjanian, A. G., Bazer-Bachi, A. R., et al. 2006, A\&A, 457, 899

Aharonian, F., Akhperjanian, A. G., Bazer-Bachi, A. R., et al. 2007, ApJ, 664, L71

Atwood, W. B., Abdo, A. A., Ackermann, M., et al. 2009, ApJ, 697, 1071

Biteau, J., \& Giebels, B. 2012, A\&A, 548, A123

Chakraborty, N., Cologna, G., Kastendieck, M. A., et al. 2015, Proc. 34th Int.

Cosmic Ray Conference (ICRC2015) [arXiv: 1509. 04893]

Chatterjee, R., Marscher, A. P., Jorstad, S. G., et al. 2009, ApJ, 704, 1689

Chatterjee, R., Marscher, A. P., Jorstad, S. G., et al. 2011, ApJ, 734, 43

Chatterjee, R., Bailyn, C. D., Bonning, E. W., et al. 2012, ApJ, 749, 191

de Naurois, M., \& Rolland, L. 2009, Astropart. Phys., 32, 231

Edelson, R. A., \& Krolik, J. H. 1988, ApJ, 333, 646

Emmanoulopoulos, D., McHardy, I. M., \& Uttley, P. 2010, MNRAS, 404, 931

Falomo, R., Pesce, J. E., \& Treves, A. 1993, ApJ, 411, L63

Gaskell, C. M. 2004, ApJ, 612, L21

Giebels, B., \& Degrange, B. 2009, A\&A, 503, 797

Gleissner, T., Wilms, J., Pottschmidt, K., et al. 2004, A\&A, 414, 1091

Hinton, J. A. 2004, New Astron. Rev., 48, 331

Kastendieck, M. A., Ashley, M. C. B., \& Horns, D. 2011, A\&A, 531, A123

Kataoka, J., Takahashi, T., Wagner, S. J., et al. 2001, ApJ, 560, 659

King, A. R., Pringle, J. E., West, R. G., \& Livio, M. 2004, MNRAS, 348, 111

Lawrence, A., \& Papadakis, I. 1993, ApJ, 414, L85

Lomb, N. R. 1976, Ap\&SS, 39, 447

Lyubarskii, Y. E. 1997, MNRAS, 292, 679

McHardy, I. 2010, in Lect. Notes Phys. 794, ed. T. Belloni (Berlin: Springer Verlag), 203

McHardy, I. M., Koerding, E., Knigge, C., Uttley, P., \& Fender, R. P. 2006, Nature, 444, 730

Nakagawa, K., \& Mori, M. 2013, ApJ, 773, 177

Narayan, R., \& Piran, T. 2012, MNRAS, 420, 604

Paltani, S., Courvoisier, T. J.-L., Blecha, A., \& Bratschi, P. 1997, A\&A, 327, 539

Priestley, M. B. 1967, J. Sound Vibration, 6, 86

Rieger, F. M., \& Volpe, F. 2010, A\&A, 520, A23

Sanchez, D. A., \& Deil, C. 2013, Proc. 33rd Int. Cosmic Ray Conference (ICRC2013) [arXiv: 1307.4534]

Sandrinelli, A., Covino, S., \& Treves, A. 2014, ApJ, 793, L1

Scargle, J. D. 1982, ApJ, 263, 835

Simonetti, J. H., Cordes, J. M., \& Heeschen, D. S. 1985, ApJ, 296, 46

Sobolewska, M. A., Siemiginowska, A., Kelly, B. C., \& Nalewajko, K. 2014, ApJ, 786, 143

Sol, H., Zech, A., Boisson, C., et al. 2013, Astropart. Phys., 43, 215

Tluczykont, M., Bernardini, E., Satalecka, K., et al. 2010, A\&A, 524, A48

Urry, C. M., \& Padovani, P. 1995, PASP, 107, 803

Uttley, P., \& McHardy, I. M. 2001, MNRAS, 323, L26

Uttley, P., McHardy, I. M., \& Vaughan, S. 2005, MNRAS, 359, 345

Vaughan, S., Edelson, R., Warwick, R. S., \& Uttley, P. 2003, MNRAS, 345, 1271

1 Centre for Space Research, North-West University, 2520

Potchefstroom, South Africa

2 Universität Hamburg, Institut für Experimentalphysik, Luruper Chaussee 149, 22761 Hamburg, Germany

3 Max-Planck-Institut für Kernphysik, PO Box 103980, 69029 Heidelberg, Germany

4 Dublin Institute for Advanced Studies, 31 Fitzwilliam Place, Dublin 2, Ireland

5 National Academy of Sciences of the Republic of Armenia, Marshall Baghramian Avenue, 24, 0019 Yerevan, Republic of Armenia

6 Yerevan Physics Institute, 2 Alikhanian Brothers St., 375036 Yerevan, Armenia

7 Institut für Physik, Humboldt-Universität zu Berlin, Newtonstr. 15, 12489 Berlin, Germany

8 University of Namibia, Department of Physics, Private Bag, 13301 Windhoek, Namibia 
9 GRAPPA, Anton Pannekoek Institute for Astronomy, University of Amsterdam, Science Park 904, 1098 XH Amsterdam, The Netherlands

10 Department of Physics and Electrical Engineering, Linnaeus University, 35195 Växjö, Sweden

11 Institut für Theoretische Physik, Lehrstuhl IV: Weltraum und Astrophysik, Ruhr-Universität Bochum, 44780 Bochum, Germany

12 GRAPPA, Anton Pannekoek Institute for Astronomy and Institute of High-Energy Physics, University of Amsterdam, Science Park 904, 1098 XH Amsterdam, The Netherlands

13 Institut für Astro- und Teilchenphysik, Leopold-Franzens Universität Innsbruck, 6020 Innsbruck, Austria

14 School of Physical Sciences, University of Adelaide, Adelaide 5005, Australia

15 LUTH, Observatoire de Paris, PSL Research University, CNRS, Université Paris Diderot, 5 place Jules Janssen, 92190 Meudon, France

16 Sorbonne Universités, UPMC Université Paris 06, Université Paris Diderot, Sorbonne Paris Cité, CNRS, Laboratoire de Physique Nucléaire et de Hautes Énergies (LPNHE), 4 place Jussieu, 75252 Paris Cedex 5, France

${ }^{17}$ Laboratoire Univers et Particules de Montpellier, Université Montpellier, CNRS/IN2P3, CC 72, Place Eugène Bataillon, 34095 Montpellier Cedex 5, France

18 DSM/Irfu, CEA Saclay, 91191 Gif-Sur-Yvette Cedex, France

19 Astronomical Observatory, The University of Warsaw, Al. Ujazdowskie 4, 00-478 Warsaw, Poland

20 Aix-Marseille Université, CNRS/IN2P3, CPPM UMR 7346, 13288 Marseille, France

21 Instytut Fizyki Jạdrowej PAN, ul. Radzikowskiego 152, 31-342 Kraków, Poland

${ }^{22}$ School of Physics, University of the Witwatersrand, 1 Jan Smuts Avenue, Braamfontein, 2050 Johannesburg, South Africa

23 Laboratoire d'Annecy-le-Vieux de Physique des Particules, Université Savoie Mont-Blanc, CNRS/IN2P3, 74941 Annecy-le-Vieux, France

24 Landessternwarte, Universität Heidelberg, Königstuhl, 69117 Heidelberg, Germany
25 Université Bordeaux, CNRS/IN2P3, Centre d'Études Nucléaires de Bordeaux Gradignan, 33175 Gradignan, France

26 Oskar Klein Centre, Department of Physics, Stockholm University, Albanova University Center, 10691 Stockholm, Sweden

27 Institut für Astronomie und Astrophysik, Universität Tübingen, Sand 1, 72076 Tübingen, Germany

28 Laboratoire Leprince-Ringuet, École Polytechnique, CNRS/IN2P3, 91128 Palaiseau, France

29 APC, AstroParticule et Cosmologie, Université Paris Diderot, CNRS/IN2P3, CEA/Irfu, Observatoire de Paris, Sorbonne Paris Cité, 10 rue Alice Domon et Léonie Duquet, 75205 Paris Cedex 13, France

30 Univ. Grenoble Alpes, IPAG, 38000 Grenoble, France CNRS, IPAG, 38000 Grenoble, France

31 Department of Physics and Astronomy, The University of Leicester, University Road, Leicester, LE1 7RH, UK

32 Nicolaus Copernicus Astronomical Center, ul. Bartycka 18, 00-716 Warsaw, Poland

33 Institut für Physik und Astronomie, Universität Potsdam, Karl-Liebknecht-Strasse 24/25, 14476 Potsdam, Germany

34 Friedrich-Alexander-Universität Erlangen-Nürnberg, Erlangen Centre for Astroparticle Physics, Erwin-Rommel-Str. 1, 91058 Erlangen, Germany

35 DESY, 15738 Zeuthen, Germany

36 Obserwatorium Astronomiczne, Uniwersytet Jagielloński, ul. Orla 171, 30-244 Kraków, Poland

37 Centre for Astronomy, Faculty of Physics, Astronomy and Informatics, Nicolaus Copernicus University, Grudziadzka 5, 87-100 Torun, Poland

38 Department of Physics, University of the Free State, PO Box 339, 9300 Bloemfontein, South Africa

39 Heisenberg Fellow (DFG), ITA Universität Heidelberg, Germany

40 GRAPPA, Institute of High-Energy Physics, University of Amsterdam, Science Park 904, 1098 XH Amsterdam, The Netherlands

41 Department of Physics, Rikkyo University, 3-34-1 Nishi-Ikebukuro, Toshima-ku, 171-8501 Tokyo, Japan

42 Now at Santa Cruz Institute for Particle Physics and Department of Physics, University of California at Santa Cruz, Santa Cruz, CA 95064, USA 


\section{Appendix A: Additional figures}

(a) H.E.S.S.
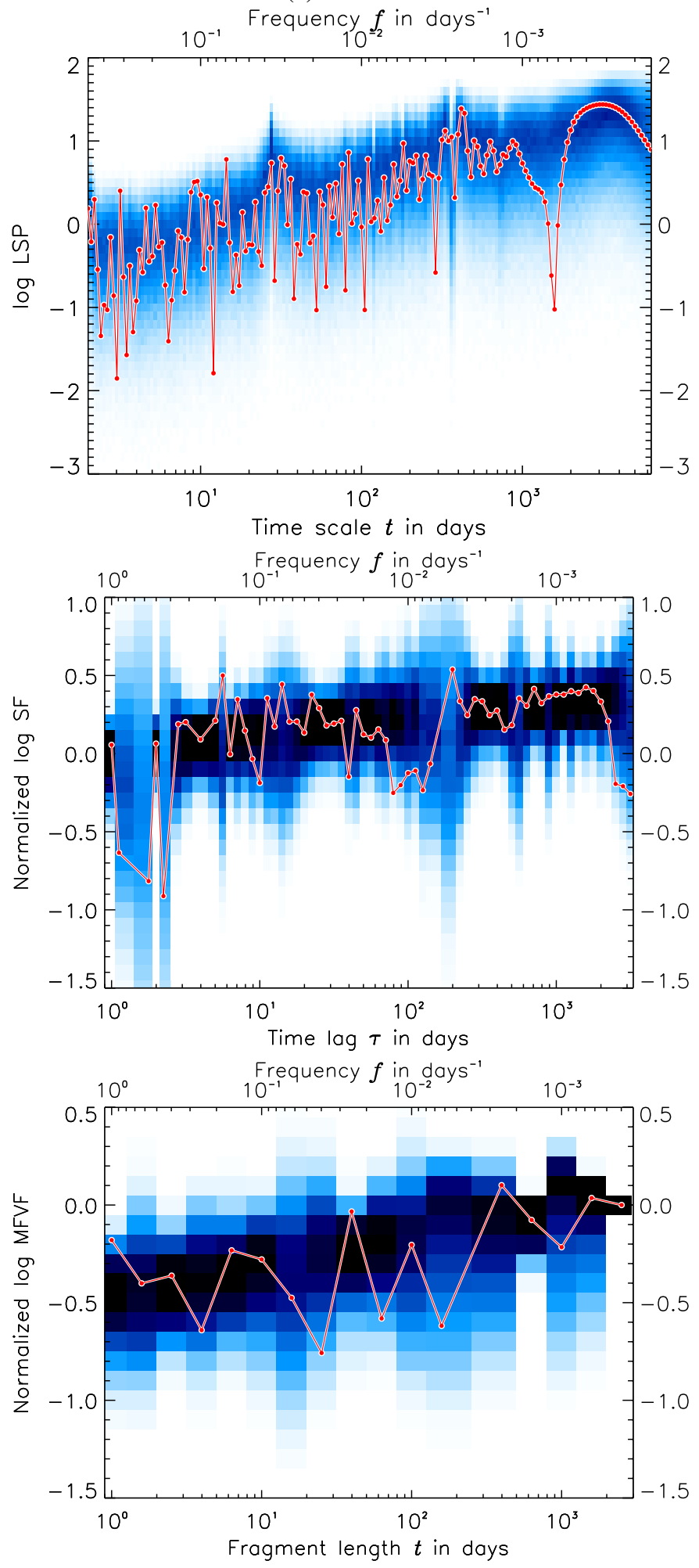

(b) Fermi-LAT
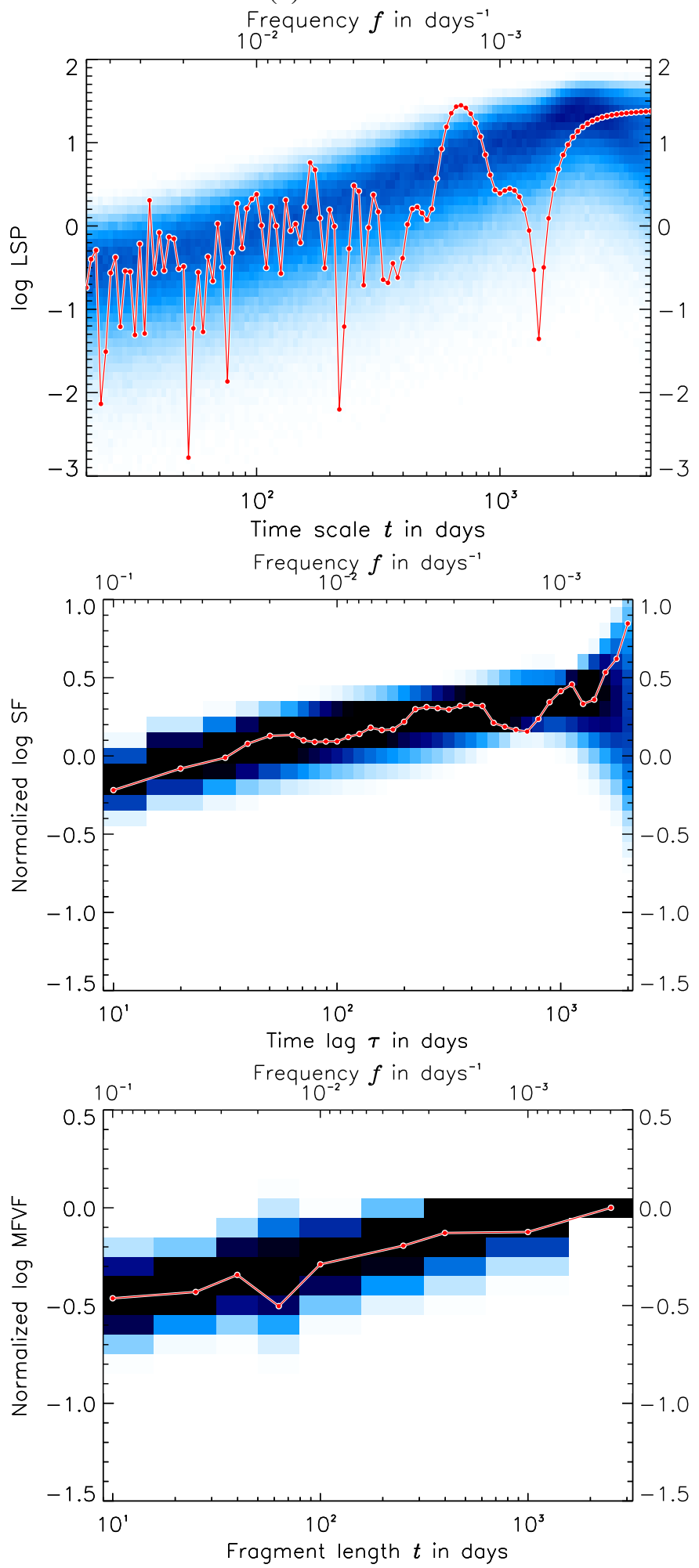
$0 \%$ $2 \%$

Fig. A.1. LSPs, SFs, and MFVFs. Left panel a) the red solid lines are the LSP (top), SF (middle), and MFVF (bottom) for the H.E.S.S. quiescent lightcurve. The best-fit PDFs of simulated LSP, SF, and MFVF values are represented by the blue histograms in color scale. Right panel $\mathbf{b}$ ) same plots for the Fermi-LAT data. 
The H.E.S.S. Collaboration: $\gamma$-ray long-term variability of PKS 2155-304

(a) H.E.S.S.
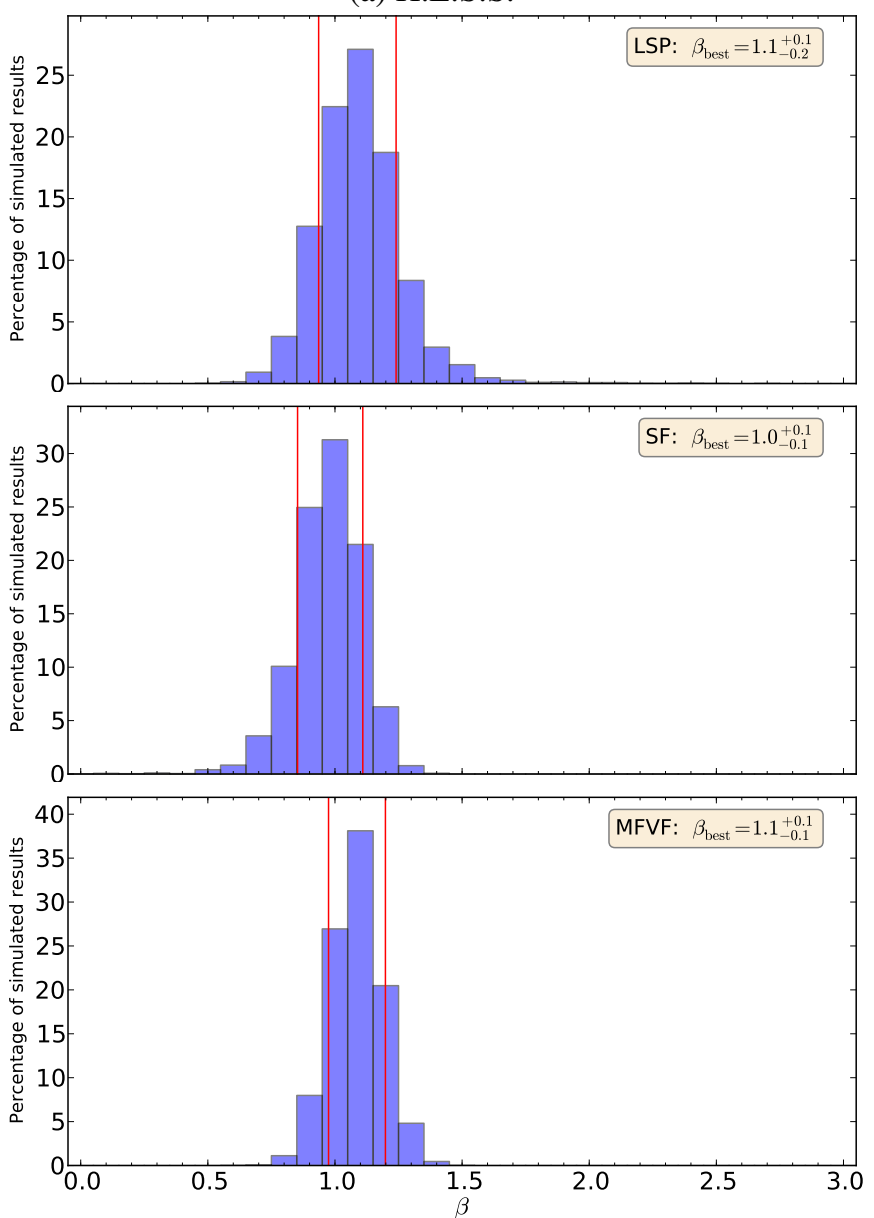

(b) Fermi-LAT
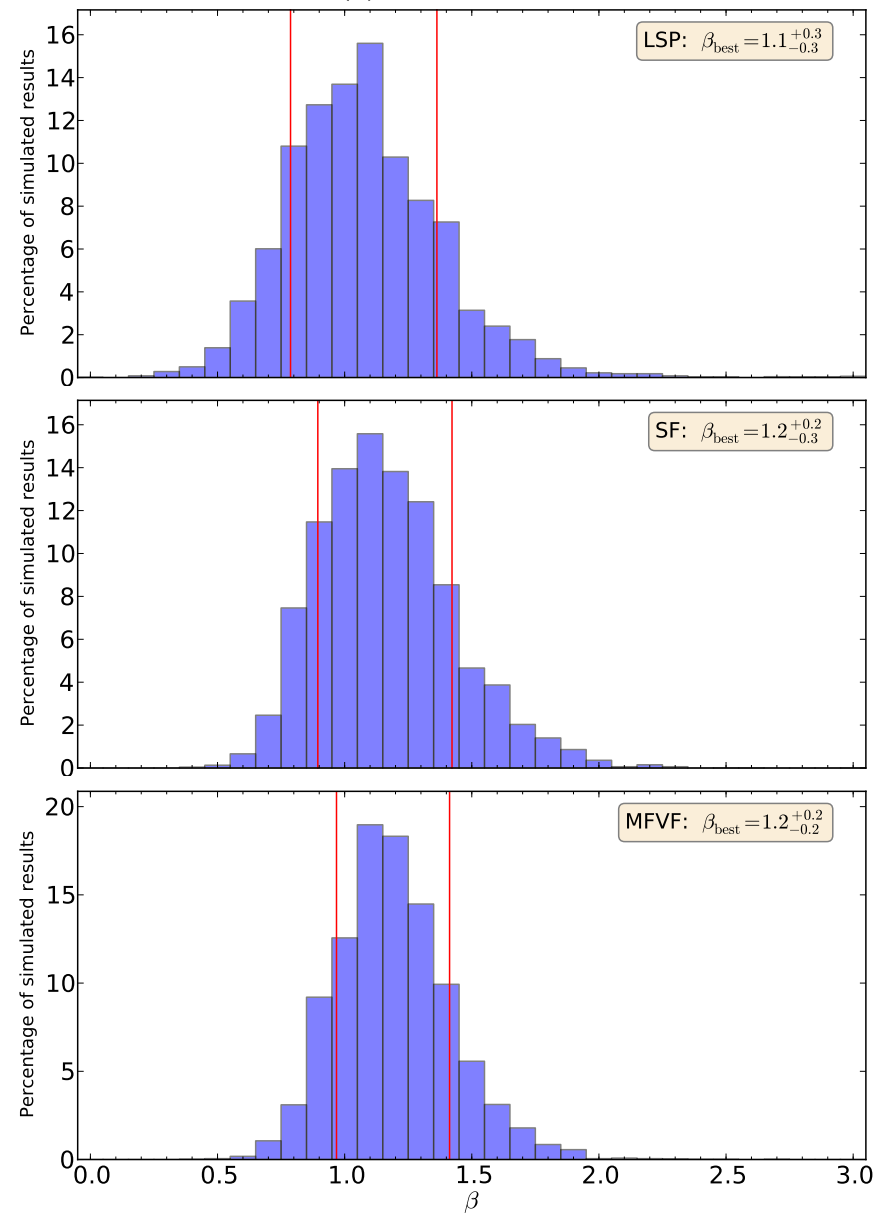

Fig. A.2. Uncertainties on the LSP, SF, and MFVF best-fit parameters. Left panel a) the histograms represent the distributions of estimates for $\beta$ for simulated H.E.S.S. light curves with the LSP (top), SF (middle), and MFVF (bottom) respectively. For the simulated light curves, the true value for $\beta$ is the best-fit value found for the H.E.S.S. light curve. The vertical red bars are the $1 \sigma$ uncertainties on the best fit obtained from the histograms by removing equal tails. Right panel b) same plots for the Fermi-LAT data. 